\title{
RAMIRO PÉREZ REINOSO: ACERCAMIENTOS Y PROBLEMÁTICAS LATINOAMERICANAS EN CHILE
}

\author{
Ramiro Pérez Reinoso: The Latin American Question and his Approach from \\ Chile
}

\section{RESUMEN}

El presente artículo aborda ciertas aristas del pensamiento del filósofo peruano Ramiro Pérez Reinoso (1901 - 19??), quien llegase exiliado a Chile en 1933. Este pensador ha sido poco leído y las referencias que hay de él son inexactas, tanto en Chile como en los textos de historia de la filosofía peruana que hemos tenido la oportunidad de revisar. Sin embargo, esta ausencia (o poco reconocimiento) ha despertado en nuestro trabajo un interés peculiar debido que, a pesar de tener un rol activo en el período de "institucionalización de la filosofía" en Chile, las problemáticas y temáticas latinoamericanistas que abordó en sus escritos no tuvieron repercusiones en la academia.

Palabras claves: Reinoso; institucionalización; barbarie; normalización.

\section{ABSTRACT}

This article presents some points of view on the Ramiro Pérez Reinoso's philosophical thought (1901 - 19??), who arrived to Chile in 1933, as a political refugee. This thinker has been little read and the references about him are not very accurate, both in Chile as in the Peruvian philosophical texts that we have had the opportunity to review. Keeping in mind his active participation in the Chilean process of "Philosophy Institutionalization" and his reflections about Latin America society, Pérez Reinoso, however, has not had the honorable place that we think he deserve in the academy.

Keywords: Reinoso; institutionalization; barbarism; normalization.

\section{UNIVERSUM}

\author{
Revista de Humanidades y Ciencias Sociales
}

\author{
ALDO AHUMADA I. \\ Mg. en Estudios Latinoamericanos, \\ U. de Chile. Docente adscrito \\ a Escuela de Trabajo Social \\ de la Facultad de Derecho y \\ Humanidades, Universidad Central \\ de Chile, Chile. \\ Correo electrónico: \\ aahumada@ug.uchile.cl
}

ORCID: 0000-0001-7354-2896

ResearchGate:

Scholar.google: Academia.edu:

\section{STEFAN VRSALOVIC M. \\ Dr. en Filosofía, U. de Chile, Chile. \\ Correo electrónico: \\ stefanvrs@gmail.com}

ORCID: 0000-0003-2463-959X

ResearchGate:

Scholar.google:

Academia.edu:

Artículo recibido el 5 de septiembre, 2019. Aceptado el 9 de septiembre, 2020.

Web: http://universum.utalca.cl | ISSN: 0716-498X - 0718-2376 


\section{BREVE MARCO BIOGRÁFICO}

El presente artículo aborda ciertas aristas del pensamiento del filósofo peruano Ramiro Pérez Reinoso (1901 - 19??), quien llegase exiliado a Chile en 1933. Este pensador ha sido poco leído y las referencias que hay de él son inexactas, tanto en Chile como en los textos de historia de la filosofía peruana que hemos tenido la oportunidad de revisar. Sin embargo, esta ausencia (o poco reconocimiento) ha despertado en nuestro trabajo un interés peculiar debido que, a pesar de tener un rol activo en el período de "institucionalización de la filosofía” en Chile, las problemáticas y temáticas latinoamericanistas que abordó en sus escritos no tuvieron repercusiones en la academia.

Por ello, el artículo tendrá como motivación ciertas preguntas que nos permitirán incursionar y matizar el pensamiento de Ramiro Pérez Reinoso. De forma inmediata, no parece tan clara la necesidad de rescatar a un pensador que ha sido poco leído, pero pareciera que sus postulados filosóficos nos permite identificar una ausencia en la filosofía chilena respecto a las temáticas latinoamericanas que plantea y los actores que incluye, como por ejemplo, los indígenas; esto deriva en preguntas como ¿por qué las problemáticas que sugiere y coloca sobre la mesa no tienen mayores repercusiones en el desarrollo de la filosofía chilena siendo que en ese momento el problema de la filosofía y cultura latinoamericana no era ajena a filósofos como Enrique Molina y Jorge Millas? Y, por último, ¿podemos ver a Ramiro Pérez Reinoso como un antecedente intelectual de las discusiones sobre la filosofía latinoamericana, en específico, sobre su originalidad y autonomía? Estas serían las preguntas principales que orientan el presente trabajo.

Lo que se ha podido recopilar hasta el momento sobre el pensador peruano es que nació en 1901 en Arica. Posteriormente, en 1923 viajó por varios lugares de Sudamérica como Argentina, Uruguay, Bolivia y Chile donde se tituló de contador, aunque nunca llegó a ejercer. A pesar de ello, su labor intelectual, al comienzo, estuvo vinculada a la poesía con una fuerte influencia vanguardista, publicando ese mismo año su poemario Pandora de 
los momentos (1923) y luego, en 1925 publicó La imitación de la luz (1926) siendo su última obra poética.

A pesar de que el poeta Federico Bolaños lo ubicó como uno de los autores que estaban renovando la poesía, Pérez Reinoso decide alejarse y comenzar a dedicarse a la ensayística y la filosofía. ${ }^{1}$ En consecuencia, en 1925 llegó a Tacna convirtiéndose en uno de los actores más importantes en el desarrollo intelectual del lugar.

Esto último se cristalizó en su participación en el primer número de la Revista Amauta $^{2}$ fundada y dirigida por Mariátegui en 1926, con la publicación de 3 escritos: un artículo sobre la revolución mexicana, que exaltaba la afirmación del estado laico frente a los propósitos de la iglesia de tener el monopolio de culto y de la educación; una reseña sobre la más reciente obra de filosofía de Mariano Ibérico, a la que caracterizó de bergsoniana; y, por último, otra reseña sobre la obra de arte plástica de Vinatea Reinoso, la cual valoró positivamente por la presencia tanto de motivos andinos como de escenas populares. Estos escritos expresan, no solo el interés personal del filósofo peruano, sino también el propósito de la Revista como lugar para las perspectivas más vanguardistas, pero, sobre todo, la orientación de las problemáticas de la llamada generación del centenario hacia un proyecto político-cultural de izquierda.

Se desconoce el motivo de su alejamiento de la Revista, y su posible ruptura personal y/o intelectual con Mariátegui, por lo que su nombre no vuelve a resonar hasta 1930 cuando comienza una labor docente en el Colegio Nacional de Varones. En 1930 se funda el diario La Nación en Tacna siendo Pérez Reinoso designado como su primer director hasta marzo de 1931. Luego, en agosto de 1931, se realiza el primer Comité Departamental del Partido Aprista en Tacna donde fue nombrado Ramiro Pérez Reinoso por el Comité Ejecutivo Nacional (CEN) del Partido Aprista Peruano (PAP) como su primer

1 Es interesante que, en Chile, Jorge Millas está pasando por el mismo desarrollo. También fue destacado como uno de los renovadores de la poesía, pero al poco tiempo ingresa de lleno a la filosofía. Pareciera que la dedicación juvenil a la poesía es más epocal que circunstancial.

2 En ella también publicaron Pablo Neruda y Amanda Labarca. 
delegado. El 6 de septiembre, Pérez Reinoso había sido nombrado secretario tesorero del Colegio Nacional de Varones y duramente criticado por el diario La Nación que pasó a manos del partido fascista Unión Revolucionaria, quienes tildaron al PAP como un partido comunista.

En estos años, se intensifica su interés por la filosofía, lo que se refleja en la obtención del grado de Doctor en Filosofía en la Universidad de San Marcos con la tesis La estética de Teodoro Lippsen 1930. Esta fue publicada dentro de su texto Ideas para una concepción histórica de la filosofia: tres estudios filosóficos (1931)

En 1932, el llamado «año de la barbarie» en Perú, el Congreso Constituyente promulga una Ley de Emergencia para reprimir a los apristas y comunistas, denominada aprocomunismo,con el pretexto de defender la democracia. De este modo, Ramiro Pérez Reinoso llega en calidad exiliado a nuestro país. Es en este punto donde el filósofo peruano llama nuestra atención. Esto se debe, en primera instancia, por el lugar que desempeñó y, en segunda instancia, por las problemáticas que planteó en sus publicaciones en Chile.

Se desconoce la fecha exacta, pero es al poco tiempo de arribar a nuestro país que comienza a trabajar como profesor de sociología en la Universidad de Chile:" "De los apristas deportados, Ramiro Pérez Reinoso fue el que más tiempo permaneció en el país de Neruda donde llegó a enseñar en la Universidad de Chile; [...]" (Kutipa, 2014: 16). En este tiempo, en Chile y gran parte de Latinoamérica, se está llevando a cabo un proceso conocido como «normalización de la filosofía», ${ }^{4}$ que refiere - a muy grandes rasgosa la instancia, a mediados de la década del 40 , en que se comienzan a crear y organizar departamentos de filosofía, revistas de filosofía, así como congresos

3 No obstante, en el Catálogo de la Escuela de verano (1935) de la Universidad de Chile es presentado como "Profesor contrata en la Escuela de Derecho de la Universidad de Chile" (15). En esta instancia, impartió un curso llamado "Problemas espirituales de la época actual".

4 Para profundizar en este concepto y sus problematizaciones, véase: Ahumada, A. (2018). Normalización Filosófica: entre filosofía y cultura. Revista De La Academia, 25, 103-133; Vrsalovic, S. (2018). Entre dos interpretaciones de la normalización de la filosofía: ¿apertura o cierre de la filosofía latinoamericana?En Filosofía e Historia de la Ciencia en el Cono Sur. (451-456). Argentina: AFHIC. 
y evento que tienen como objetivo el que los filósofos latinoamericanos se conozcan y se lean entre sí.

Este momento es particularmente importante, debido a que, por una parte, la filosofía se hace parte del quehacer cultural y es una actividad común a otras como la literatura; y, por otra, coloca a la filosofía como un quehacer que debe ser realizado dentro de la academia para poder tener el rótulo de filosofía. Es por ello, que la «normalización de la filosofía» va de la mano con la institucionalización de la filosofía. Y en Chile, esta última tiene una de sus expresiones más claras en la creación de la Sociedad Chilena de Filosofía, que, sin pertenecer a una institución en particular, agrupa a todos o, al menos, los más conocidos, pensadores que abordan problemáticas filosóficas, y se establece como el lugar que determina qué es y qué no es filosofía, llegando, incluso, a confeccionar el programa de filosofía para los Liceos.

Es en este ambiente donde se instala Pérez Reinoso y con una participación bastante activa. El 29 de julio de 1948, en la Biblioteca Nacional, se conforma la Sociedad Chilena de Filosofía, siendo su Presidente Enrique Molina y el pensador peruano ocupando el cargo de Secretario de Difusión ${ }^{5}$ hasta 1953 que se produce un recambio en la directiva de la Sociedad. ${ }^{6} \mathrm{La}$ Sociedad tiene como propósito, según sus estatutos, el promover, impulsar y difundir los estudios filosóficos, y para ello se propone realizar sesiones de estudios periódicas, así como organizar congresos, cursos, publicaciones, etc.

La primera sesión de estudios estuvo a cargo, justamente, de Ramiro Pérez Reinoso, quien disertó «Jonathan Edwards en el Pensamiento Colonial de Estados Unidos», y "se refirió a los antecedentes históricos, a sus obras y a la importancia que estas doctrinas tuvieron en el desarrollo intelectual de los

5 Hay una pequeña confusión, ya que en el primer número de la Revista de Filosofía (1949) se señala que Pérez Reinoso ocupará el cargo de Secretario de Publicaciones y Mario Ciudad el de Difusión (p.96), sinembargo, en la última página sale un organigrama donde los puestos están al revés. Creemos que el error está en la página 96 ya que Mario Ciudad es quien ocupa el cargo de Director de la revista, teniendo más sentido su función como Secretario de Publicaciones que de Difusión.

6 Cabe señalar que Enrique Molina mantendrá su cargo de presidente. 
Estados Unidos en el período colonial.” (Vidal, 1949: 96). ${ }^{7}$ Podemos especular, a partir del cargo que ocupó, así como de la responsabilidad de encargarse de la primera sesión de estudios de la Sociedad, que no era una figura desconocida en el ambiente académico del país y que podría ser considerado, en primera instancia, como un representante claro de la «normalización filosófica» chilena.

Este último punto cobra mayor relevancia cuando se estudian las obras que publicó en Chile, ya que no necesariamente está pensando desde un modelode filosofía institucionalizada, en el sentido que no responde directamente a la producción que se está realizando en ese momento. ¿Es posible, entonces, hablar de autonomía filosófica a pesar de pertenecer a la institucionalidad filosófica? Es una pregunta más que pertinente frente a la producción de Ramiro Pérez Reinoso. Dentro de la producción que realizó en nuestro país ${ }^{8}$ podemos encontrar un artículo en la Revista Atenea (1932) «El problema de la autonomía espiritual de la América Latina» que tiene como objetivo contribuir al desarrollo y esclarecimiento de los problemas culturales de Latinoamérica. En esta misma línea se encuentran el libro Mensaje sobre el destino de la cultura en América Latina (1947). También publicó la traducción de la autobiografía de Rudyard Kipling: Escenas de mi vida (1937), la traducción de Bolivar de Phullis Marschal y John Crane (1938) y Trayectoria de un soldado (1940).

Los dos primeros textos mencionados tienen un fuerte carácter latinoamericano ya que persiguen problematizar lo que se está realizando y en qué condiciones se puede hablar de una autonomía filosófica. No obstante, antes de entrar en su problematización, y ahondando en este poco reconocimiento, podemos señalar que esta perspectiva tampoco es mencionada por sus coetáneos. Por ejemplo, su compatriota David Sobrevilla solo lo nombra en su texto La filosofía contemporánea en el Perú como uno de los representantes

7 En la Revista solo publica en el primer número dos notas cronológicas sobre Mons. Eduardo Escudero Otárola y Dr. Guillermo Mann,(113-116).

8 Como mencionamos anteriormente, ya había publicado poemarios en Perú, pero, además, en 1931 publicó en Lima: Ideas para una concepción histórica de la filosofía (1931) que contienen su tesis doctoral como dos textos sobre Spengler. 
del momento que denomina "La reacción espiritualista (1900-1920)" que se articulaba en la Universidad de San Marcos: "El espiritualismo tiene en el Perú fundamentalmente dos actores: el bergsonismo [...] y la generación arielista, $[\ldots]$ Además hay otras figuras cercanas o antagonistas y una segunda generación de espiritualistas (conformada por Manuel Argüelles, E. Barboza y R. Pérez Reynoso)" (1996: 175).

Por otra parte, un pensador peruano consagrado a estas alturas como Augusto Salazar Bondy no le dedica más que una línea a nuestro pensador en su texto La filosofía en el Perú para darlo como ejemplo al tránsito de la influencia filosófica francesa a la alemana: "Por su parte, Ramiro Pérez Reinos adoptó y divulgó el pensamiento de Spengler.” (1967: 109) En este tránsito también nombra a Manuel Argüelles y Enrique Barboza, pero no menciona la problematización en torno a la autonomía de la filosofía latinoamericana.

Por último, en Chile también es poco mencionado, las escasas referencias que hemos encontrado son de Roberto Escobar en su libro El vuelo de los búhos, en la que solo hace una lacónica reseña más un par de citas dispersas de nuestro autor; el otro autor, que lo cita de manera más breve aún - solo cuatro líneas- es Enrique Molina en su texto La filosofía en Chile en la primera mitad del siglo $X X$. Más de eso prácticamente no hemos hallado.

Desde esta arista, o, en otras palabras, desde este poco reconocimiento, es que nos proponemos instalar una propuesta de discusión de los planteamientos de Reinoso pero que abordan los problemas latinoamericanos, más específicamente, la posibilidad de una filosofía latinoamericana. Estos planteamientos no han sido abordados por los autores que hemos mencionado, por lo que nuestra perspectiva es más bien una tentativa, un posible acercamiento a las discusiones teóricas que se pueden articular a partir de lo dicho por el pensador peruano. Es por ello que el artículo no tiene y no pretende tener un carácter conclusivo, sino más bien, de apertura a nuevas formas de entender los momentos históricos donde se ha discutido sobre filosofía latinoamericana. 


\section{APROXIMACIONES AL LATINOAMERICANISMO DE PÉREZ REINOSO}

Una de las cosas que más nos ha llamado la atención del pensamiento de Pérez Reinoso, es la actitud y los esfuerzos que empeña en pos de construir un verdadero programa emancipador del ser humano latinoamericano. Sus esfuerzos intelectuales en reiteradas ocasiones parecieran invitarnos a buscar la siempre anhelada independencia cultural. Bajo sus términos, los esfuerzos deben apuntar a las formas y caminos que, asumiendo las dificultades del asunto, nos lleven hacia la «autonomía espiritual». Para él, se hace necesario tener que pasar por ciertas «etapas» de desarrollo cultural que se nos hace importante mencionar, pues, la filosofía ha de ir acompañada de múltiples expresiones del ser humano que apunten hacia las necesidades particulares de cada región. Así, tendríamos la oportunidad de ver, de cierto modo al menos, emerger un pensamiento con una carga de originalidad que, a juicio del peruano, no ha podido apreciarse más que en ciertos tenues destellos de corta duración; uno de ellos: Simón Bolívar.

Para desarrollar las reflexiones del filósofo peruano, partiremos analizando cuáles serían las condiciones en las que la cultura latinoamericana se encontraría en el presente del autor, las cuales, para decirlo de entrada, serían propias de pueblos que, según Pérez Reinoso, recién comenzarían a encontrar ciertos destellos que nos darían la apertura hacia el camino propio.

No obstante, nos es indispensable dar atisbos de la definición de filosofía que utiliza Ramiro Pérez Reinoso. En el libro Mensaje sobre el destino..., el filósofo peruano inicia diciendo que "La filosofía debe ser la historia metafísica del mundo; la dirección y valoración de la alta odisea del espíritu" (1947: 7). Es decir, la filosofía será uno de los derroteros principales, si no el más importante, para la forja de una historia que debe estar intencionada hacia un fin particular: dar las principales directrices para suplir las necesidades propias de los pueblos latinoamericanos; las cuales no podrían ser satisfechas desde el ejercicio del estudio y copia del pensamiento europeo. En otras palabras, la 
filosofía es guía e iluminación.

Esta concepción de la filosofía es una idea que puede ser rastreada en escritos anteriores al libro mencionado. Por ejemplo, en el libro Ideas para una concepción..., podemos leer que la filosofía es como "un contenido natural afectado de esperanza, de historia, de porvenir; como dirección política en el mundo de las fuerzas instintivas del espíritu" (1931: 78); o bien, "la filosofía es, pues, un gran viaje por el país de la bruma universal. Un sistema claro del universo en cuanto al hombre, que con tanta frecuencia exije [sic] nuestra mente, es una lámpara" (1931: 79). Así, vemos que la filosofía, tal como ya hemos anunciado, debe ser expresión del desarrollo espiritual de los pueblos, es parte axial de los engranajes que mueven la historia, y lo es debido a su alta conciencia de la realidad.

Para el avance de tales ideas para el desarrollo de nuestra «autonomía espiritual», es necesario levantar toda una cultura que logre unificar los pueblos de nuestra América, punto que, a nuestro juicio, se presenta como el más difuso de resolver desde las ideas de nuestro autor. Lo que sí nos indica Pérez Reinoso, es que, sin un claro desarrollo de nuestra economía y ciencias exactas, difícil será levantar una cultura fuerte que logre despegarse tanto de la naturaleza que sobreabunda como del pensamiento subyugado a las directrices de las novedades europeas.

En una conferencia realizada en la Universidad de Chile el 12 de mayo de 1932, la cual lleva por título El problema de la autonomía espiritual de la América Latina, notamos que para Ramiro Pérez Reinoso, la América antes del inicio de lo que, siguiendo a Quijano, denominaremos «colonialidad», ${ }^{9}$ estaba

9 Por «colonialidad» entenderemos un nuevo "patrón de poder de vocación mundial" que surge con el inicio de los procesos de conquista y colonización de América por parte de los europeos. Según nos dice Aníbal Quijano, “América se constituyó como el primer espacio/ tiempo de un nuevo patrón de poder de vocación mundial y, de ese modo y por eso, como la primera id-entidad de la modernidad. Dos procesos históricos convergieron y se asociaron en la producción de dicho espacio/tiempo y se establecieron como los dos ejes fundamentales del nuevo patrón de poder. De una parte, la codificación de las diferencias entre conquistadores y conquistados en la idea de raza, es decir, una supuesta diferente estructura biológica que ubicaba a los unos en situación natural de inferioridad respecto de los otros. Esa idea fue asumida por los conquistadores como el principal elemento constitutivo, fundante, de las 
sumida en el mundo natural, o bien, tal como nos lo dice nuestro autor: "Al americano antiguo, sin mecánica y sin química, — sin técnica - la naturaleza le imponía sus moldes y él los llenaba con su vida y sus instituciones y su conducta política" (1932: 121). En relación a los grupos que, de una u otra forma, fueron desligándose del mundo natural, sobreponiendo a este mundo lo que podríamos denominar de forma laxa «cultura», Pérez Reinoso indica que: "en los casos de mayor espiritualidad el hombre trascendentalizó la fauna, la flora y la tierra agreste en formas religiosas y míticas, ya dentro de la fe, ya dentro de la política" (1932: 121). Es decir, los grupos humanos que habitaban esta parte del planeta aún tenían su centro articulador anclado al mundo religioso y mítico y, tal como ya ha sido anotado, sin una separación clara entre los hombres y mujeres y la naturaleza; idea característica del hombre moderno que Pérez Reinoso valida como necesaria para una genuina madurez del espíritu. Sin embargo, nuestro autor identifica las denominadas "altas" culturas, para diferenciarlas de los grupos humanos que no poseían vida urbana tan intensa, bajo un momento de desarrollo el cual estaría a las puertas de la escisiónser humano y naturaleza, lo que habría sido efectuado desde una maduración de cauces propios, y que les daría un estatus de autonomía mayor que los americanos de inicios del siglo XX. En palabras de Pérez Reinoso, vemos el panorama de estos grupos bajo las siguientes palabras:

Los aztecas, mayas y quechuas estaban, pues, en el final de un proceso evolutivo de siglos, y en este sentido era más civilizados que los americanos de hoy, que viven más para el porvenir que para el pasado y forman pueblos en crecimiento.

Entonces los americanos tuvieron una autonomía espiritual. Los progresos de su última etapa fueron creados por ellos y para ellos. No importa cuáles hayan sido los orígenes de sus diversas culturas; lo real es que ya habían cumplido todos los pasos de su programa de vida y que sólo les restaba medrar y conservar. Su autonomía era el resultado de su crecimiento biológico que había llegado a cuajarse en la civilización vigilante del grupo de hombres en que descansaba el gobierno teocrático. (1932: 121)

relaciones de dominación que la conquista imponía" (Quijano, 2000: 202). 
A medida que avanza la conquista del mundo de los pueblos originarios por parte del mundo occidental, Ramiro Pérez Reinoso nos dice que los españoles logran construir una sociedad divorciada de la naturaleza, la cual, vía encomiendas, logra llevar una vida en las urbes donde aflora la ociosidad, o, como bien nos dice el autor, entregándose "a las complacencias de la vida de las ciudades" (1932: 122). Pues, su principal preocupación radicaba en el inspeccionado de tierras y pueblos de indios que le habían sido entregados como quien entrega ganado. Este es el argumento que nos da nuestro autor para decir que:

Hay, pues, en la sociedad extranjera una evasión de la naturaleza. Por eso logra fielmente repetirse en la vida de las ciudades el tono, la ostentación y la psicología de la vida española peninsular. La sociedad española vivió trasplantada durante tres siglos. (1932: 122).

Aquella forma de vida, la cual toma personalidad característica de los pueblos del nuevo mundo al ver en franco retroceso a las culturas originarias producto de la conquista, produce una suerte de elipsis histórico. Aquel vacío o bien estancamiento del desarrollo cultural de las sociedades que empezaban a nacer en la América española era producto de una vida licenciosa que impidió un salto cualitativo hacia el despegue de una autonomía, tal como nos lo dice nuestro autor:

Sensualismo, ociosidad, escasez de preocupaciones. Entonces se comienza a dar importancia a los hechos frívolos a los que la curiosidad y la fantasía transforman en temas de los que no puede prescindir el que quiere vivir al día. (1932: 121)

Luego, la historia es más bien conocida, aunque no menos problemática. Una rebelión contra el poder gobernante de la metrópoli trajo un cambio en los sistemas políticos, tal como lo va relatando Pérez Reinoso. Esta emancipación del orden colonial es una de las primeras puertas, desde el proceso de "encubrimiento" de América, en donde podría verse reflejado el ansia de una «autonomía espiritual», pues al desprenderse del poder que subyugaba a los pueblos americanos, se produce la reflexión y el cultivo de un espíritu que 
busca los caminos propios:

Los ideales republicanos y democráticos de entonces eran el anticristo de toda civilización o toda catalepsia social. Es por esto que puede señalarse a Bolívar con su radicalismo republicano como el primer campeón de nuestros esfuerzos por una cultura autónoma. En aquella época y en el escenario de América ser republicano significaba decretar nuestra propia personalidad. (1932: 122)

Para Ramiro Pérez Reinoso, las primeras décadas que abren nuestro siglo XIX, las primeras tres para ser exactos, serían las más representativas en relación con la búsqueda de lo propio. Desde su óptica, aquellas tendrían una carga americanista que no poseería ninguna otra del siglo, pues la ilustración europea aún no ha copado las conciencias ni tampoco, según su criterio, habría real contenido histórico. Lo que encontraríamos sería un espíritu juvenil cargado de ideales, los cuales comenzarían a difuminarse a medida que las elites de los distintos países tienen cada vez más facilidades para viajar e ilustrarse en el viejo mundo. Es en este momento cuando los distintos pensadores de América borrarían con el codo lo que habían ya escrito con sus manos. El proceso de "aprendizaje" de los modos y formas de pensamiento europeos, es decir, la cultura occidental, dejaría un cierto ideal que alcanzar. Ideal que, por cierto, tiene grandes distancias frente una realidad americana que aún viviría en una suerte de barbarie y la desorganización política propia de "pueblos nuevos". En virtud de lo dicho, Ramiro Pérez Reinoso nos dirá las siguientes palabras:

Después cuando las clases ilustradas y pudientes pueden con facilidad visitar Europa y recorrerla como un placer entonces comienza la europeización verdadera, esto es, cuando el alma popular comienza a ver lo importante y perfecto y bello sólo en lo que tiene algo de los grandes países civilizados. (1932: 122)

Tal como nos lo dice el pensador peruano, la europeización será en su mayor parte una planificación, un plan político emanado de los gobiernos. El caso de Chile y Argentina serían ejemplares; tal como lo relata Pérez Reinoso, tomarían a modo de ensalmo las palabras de Sarmiento que separarían y estipularían lo qué sería la civilización y la barbarie. 
Aquel intento transformador, en un sentido estrictamente occidental, de nuestra cultura será, entonces, una suerte de condena para los proyectos de autonomía intelectual, pues despreciarían la naturaleza y su potencia como fuerza creadora: el más auténtico reservorio de autenticidades. La naturaleza, desde el punto de vista de la civilización, no sería más que un conjunto de riquezas a explotar. La naturaleza, entonces, sería vista como un mero objeto comercializable cuando en realidad guardaría la fuerza desde donde debiésemos afirmarnos para comenzar a caminar a paso propio. El salto de la historia, la cual debe ser historia dirigida y no un mero camino azaroso, debe tener como condición indispensable nuestra naturaleza y las razas que habitan en ella. En relación con la naturaleza, las palabras de Pérez Reinoso nos parecen elocuentes, pues ahí es donde podemos apreciar de manera más clara su concepción "decolonizadora":

De una valorización solo económica nuestra naturaleza puede pasarse a una valorización puramente comercial y entonces no podría estar garantizada aún la integridad física del continente. Hay que dar, pues, a esta concepción del factor naturaleza, algunas raíces psíquicas más profundas que la apreciación intelectual. La imagen de la mina, del fundo, de la caída de agua, de la tierra colonizable, debemos enmarcarla en la idea intuitiva de la gran patria. En esa idea están todos los contenidos psíquicos y sociológicos que deben desarrollarse en nuestra aspiración americanista, cuyos caminos, por eso, no pueden ser otros que los del arte, de la literatura, de la modalidad política y de la exaltación racial, es decir, de la incorporación de las razas americanas auténticas en las responsabilidades del comando de nuestros pueblos. Ellas que están más en contacto con la naturaleza y que la sienten con mayor fuerza en el eje mismo de sus almas son la garantía mayor de nuestra autonomía espiritual y política. (1932: 124)

Con relación al levantamiento de las razas "auténticamente" americanas, si es posible determinarlas de ese modo, Pérez Reinoso no cae en esencialismos. Según nos dice en Mensaje sobre el destino..., "el indio es tradicionalista en lo que hay de ausente en su alma porque ni siquiera alcanza a vivir en un vago sonambulismo el recuerdo de las antiguas culturas de América" (1947: 19); por lo tanto, propone el filósofo peruano, nuestras culturas originarias en vez de un intento por irse a sus profundidades culturales, cosa imposible debido al borramiento de sus formas de ver el mundo, deben darse una vuelta por otras 
culturas para así "amnistiarse psicológicamente" (1947: 19).

Respecto al estatus de humanidad que poseería el hombre natural americano, es decir, su grado de inferioridad o superioridad en relación con el ideal de hombre y mujer que brinda la actitud colonial del ver, Pérez Reinoso da una interesante apreciación: "Declarar científicamente inferior a una raza es cuando más afirmar un hecho presente pero no una ley social del pasado ni del porvenir" (1947: 27). Entonces, podemos afirmar que en el filósofo peruano hay una reivindicación identitaria que perfectamente podríamos catalogar de no esencialista, pues no identifica lo propio con lo celosamente originario de aquí, ni tampoco busca una comparación racial que apele a jerarquías propias de un pensamiento europeo. Tampoco busca apegarse al pensamiento occidental así como así, lo que nos parece de suma importancia destacar frente a otros intentos de construcción identitaria que se están desarrollando en el continente. Tal como Martí nos dijera: "Ni el libro europeo, ni el libro yankee, daban la clave del enigma hispanoamericano" (1986: 127), Pérez Reinoso apuntará a la misma idea: "sin profundidad ni intuición para el examen sociológico y con criterios parasitarios respecto de escuelas europeas hechas para el uso de Europa [...] han expresado su pesimismo por el porvenir de nuestro continente" (1947: 24). Luego continuaría con una idea en franco alejamiento del pensamiento positivista que aún deambularía por los recovecos intelectuales de nuestra América y que vendría a siempre validar nuestra inferioridad:

Por fortuna el ensanchamiento de los horizontes científicos y los nuevos puntos de vista deducidos de la crítica histórica contemporánea están barriendo las ropavejerías retardatarias, los dogmas de estrechez positivista, los dictámenes de jurisperitos de las ciencias con mentalidad de "época de los descubrimientos". (1947: 24)

O bien como dice en otro pasaje del mismo texto:

Resulta estúpido hablar ahora de razas atrasadas al referirse a pueblos que de algún modo actúan ya en medio de la civilización, pues en este caso hay sociedades y no razas atrasadas, teniendo su origen este atraso en los desequilibrios y las injusticias de la organización social y económica. (1947: 69) 
Frente a este desafío de no caer en esencialismos, pero tampoco en la pasividad frente a lainfluencia europea, el rol de los intelectuales es fundamental. Para Pérez Reinoso debe ocurrir una suerte de autoconciencia en ellos para así lograr establecer las bases de una historia dirigida. Aquella dirección tiene la particularidad, podríamos deducir, de coger una cierta actitud que se rescata de la cultura occidental, pero con la intención de reivindicar y levantar las particularidades propias de los hombres y mujeres que habitan este continente en su relación con su tierra y su cultura. Así, estos grupos, que pasarían a ser, de cierto modo, "intelectuales orgánicos", tendrán la responsabilidad de instalar las conductas necesarias que potencien los espacios en donde aún podrían hallarse signos de originalidad. En sus palabras: "Para la organización de los factores y los elementos dispersos se reclama la intervención de la inteligencia" (1947: 47). Esta inteligencia deberá potenciar, sabiendo cómo tomar lo provechoso y dejar a un lado lo que estorbe, lo que queda de bárbaro en el continente; pues es ahí donde se encontraría el potencial necesario para desprenderse del pensamiento que gravita en torno a Europa.

El pensamiento "occidentalizante" sería propio delos grupos cultos del continente, los cuales al pensar desde un lugar el cual no es el suyo, han perdido la capacidad de conectarse con su propio entorno. En esto Pérez Reinoso tiene una postura interesante: por un lado, los grupos más cultivados y versados en lo que a cultura occidental respecta deberán arraigarse a sus contextos locales e impregnarse de su realidad; y, por otro lado, los indígenas sumidos en la naturaleza deberán realizar el salto hacia una reflexión que los lleve a sobrepasar los límites que les impondría el vivir imbuidos en el mundo natural:

La realidad sociológica que se le presentaría a la inteligencia con caracteres de gran problema es que en los sectores europeizados no hay atisbos de originalidad para esperar por ahora una filosofía y una cultura propias, y en los sectores bárbaros no hay ya tradición viviente de las culturas desaparecidas para esperar impulsos nuevos y originales mediante su transformación. Pero la Inteligencia tiene que comprender que la originalidad y sus elaboraciones sociológicas sólo pueden surgir del fondo del alma bárbara, de la sociedad del colono psicológico. Por lo tanto, la misión de la Inteligencia en los campos político y social adquiere caracteres realmente grandiosos. (1947:72) 
En otro pasaje, podemos apreciar ideas similares:

El latino-americano culto o medianamente cultivado ha seguido un plan de economía de esfuerzo al aceptar la imitación y la copia de cosas europeas y norteamericanas antes que darse al "abrazo de la tierra" y después de una peregrinación religiosa y estética resultar habitando su propio estilo de vida. Pues la diferencia es tal que mientras el indígena sedentario necesita liberarse de la tierra el hombre cosmopolita de las grandes ciudades haría bien en vincularse al agro y el paisaje. (1947: 73-74)

Como se ha señalado, el apego a la naturaleza y la distancia que el ser humano "natural" de América tendría del mundo urbano, daría la oportunidad de encontrar en esa particularidad una originalidad reflexiva que sería difícil encontrarla en otros sitios. En otras palabras, replicando las ideas del filósofo peruano, el hombre bárbaro sería un crisol de pensamientos de carácter particulares. Ahí es donde podría hallarse una originalidad que ha sido difuminada y perdida gracias al excesivo vínculo que poseen los grupos dirigenciales de las distintas urbes latinoamericanas y, claro está, por el colonialismo que cercenó las memorias de los pueblos originarios de estas tierras:

El hombre bárbaro, que es el más fecundo valor sociológico para construir la América futura, debe ser el punto central de los planes de todo pensador político y filosófico y de todo educador constructivo. El hombre bárbaro latino-americano debe continuar en diálogo con la tierra y al margen de toda civilización social trasplantada que se traduce en el imitador en frivolidad y afeminamiento. Su condición de provinciano psíquico, de montañés con ambiciones organizadas y fuertes y una ética de progreso y ambiciones nacionales y continentales serán las bases humanas más sólidas del futuro mundo latino-americano. (1947: 74)

Ramiro Pérez Reinoso continuará señalando que "El hombre bárbaro es la provincia sociológica de toda nuestra América. Hay que alentar su continuidad histórica y disciplinar y orientar sus pocas pero claras pasiones elementales" (1947: 74). Por lo tanto, aquel hombre y mujer deberá ser "el motivo humano más importante de la nueva política y la nueva educación." (1947: 74). Esta defensa y, creemos, reivindicación de lo bárbaro o de la barbarie es particularmente interesante, debido a que toma un elemento que 
le permite colocarse en un punto teórico intermedio pero que no por ello es menos fructífero. De este modo, trata de congeniar el espíritu racional con las pasiones elementales propias del ser humano subsumido en la naturaleza.

Es por ello, que la «Inteligencia», como la denomina el filósofo peruano, si algo debe rescatar de la intelectualidad europea, debe ser la actitud de cómo pensar para dirigir planificadamente el devenir de una cultura que, desde su óptica, de a poco comienza a tomar fuerza y dirección. Más que eso no es mucho lo que podría apropiarse, ya que el cultivo en la cultura europea más que cualquier otra cosa llevaría a tomar distancia de la realidad de donde justamente debería arraigarse nuestra intelectualidad para así levantar un pensamiento filosófico que tenga algo que decir, y no agotarse en una repetición más bien estéril:

\begin{abstract}
Mientras más alta sea la ilustración de los grupos que cultiven la filosofía la influencia de la naturaleza y la provincia latino-americana será menor en ellos, pues la ilustración de estos grupos fatalmente tendrá una formación psicológica e intelectual de tipo europeo lograda en los libros, los viajes y los contactos de toda especie con la Europa occidental.

Sin embargo, esa condición no tendrá un significado pesimista porque el pensamiento filosófico no sólo lo hacen en general y lo harán los intelectuales puros y los eruditos en filosofía pasada. El pensamiento filosófico puede ser el fruto de los idealistas, de los hombres que frente a la vida y a la historia de América tomen una determinada posición espiritual y sentimental. En este caso el pensamiento filosófico puede ser una elaboración del espíritu bárbaro. (1947: 78)
\end{abstract}

El pensamiento filosófico que cargan y elaboran los distintos grupos humanos, no es característico ni exclusivo de naciones de la, si es que se puede llamar así, «vieja tradición intelectual». Se agrega a esto que, tampoco, la filosofía es "el tramo final de una activa y sinuosa línea de progreso" (1947: 81). Es más, Pérez Reinoso nos advierte que el pensamiento filosófico que mayormente se elabora en las épocas de "gran civilización", como las denomina, suelen ser "etapas hipócritas del progreso" (1947: 81). Esta apreciación tiene su raíz en que las sociedades que están en una etapa ya "madura", según los cánones propios de la época y arrimándonos a lo que 
Dussel alguna vez denominó falacia desarrollista,${ }^{10}$ el pensamiento filosófico tiende a ser más bien carente de proyectos constructivos, o, tal como dice Pérez Reinoso: "la corriente de la vida es periférica y circular" (1947: 81). Es por eso que, los distintos pensadores y creadores de pensamiento filosófico, repararán en temáticas tendientes a rebuscar en las interpretaciones y en la búsqueda de sistematizaciones y creación de sistemas que poco tienen de energía constructiva; más bien se quedan circulando en ideas que poco sentido tienen en la construcción concreta de sociedades. Esta interpretación puede explicarse desde la argumentación de que los países de "alto progreso", o "países ricos", al llegar a una suerte de equilibrio social tienen la oportunidad de reflexionar en torno a temáticas que se distancian del pensamiento constructor y cuestionador de los proyectos sociales. Los países viejos tienen poco futuro al que mirar, pues su preocupación recae en mantenerse en donde están: tienen otras preocupaciones y la filosofía por lo mismo se apertura hacia otras sensibilidades de corte más bien conspicuo, disminuyendo la producción de pensamientos de corte materialista (constructor de proyectos). Esta idea se ve reflejada en las siguientes palabras:

Es muy común creer pensamiento filosófico a la nutrida discusión de doctrinas y escuelas por los profesores y eruditos, al ir y venir de las nuevas interpretaciones acomodadas a todo tipo de desorientación de los espíritus. Eso es sencillamente el deporte intelectual a que se entrega una sociedad civilizada que cuenta con ocios para ellos [...] La erudición intelectualista asoma como síntoma de vejez durante aquellos grandes conversatorios que hace la humanidad en cada remanso de su marcha. Se hace entonces de la filosofía una institución canónica obediente a escuelas, normas y principios, y como no hay capacidad creadora queda, al menos, el consuelo de la habilidad imitadora. (Pérez Reinoso, 1947: 82-83)

Continuando con esta idea, el filósofo peruano indica que los momentos de grandes sistemas en donde se busca sentido transcendental, es en el principio de un proceso de civilización, "antes del momento en que

10 Enrique Dussel en las conferencias reunidas en el libro titulado 1492 El encubrimiento del otro, entiende por falacia desarrollista "una posición ontológica por la que se piensa que el "desarrollo" que siguió Europa deberá ser seguido unilinealmente por toda otra cultura" (1994: 13). 
cada pueblo comienza a servirse el gran banquete de las conquistas ideales y materiales que realizó en su época de crecimiento" (1947: 83). Por eso es que el pensamiento emanado del «espíritu bárbaro» tiene lógicas de originalidad, ahí se encuentra un espíritu que apunta a lo creativo, apunta a una filosofía auroral $^{l l}$ que, a diferencia de las filosofías que reflexionan en torno a lo acaecido, tiene la responsabilidad de construir futuros posibles para el grupo humano al cual toma como motor de su quehacer intelectual.

Con estas ideas ya establecidas, no es difícil entender el porvenir que anhela Ramiro Pérez Reinoso para el pensamiento que pudiese surgir de los grupos humanos apartados del mundo urbano en nuestra América. Ahí es donde, desde su perspectiva, hay que poner las esperanzas para encontrar algún pensamiento filosófico que pueda llevar algún título de originalidad:

La filosofía más emocionada y honda, que es casi siempre la menos erudita y compleja, coincide con el momento bárbaro del espíritu. Entonces el pensamiento filosófico es más fuerte y original y es como la raíz elabora de inmediato las primeras energías del mundo. Por lo menos en esta América, que exige una organización de los principios y una orientación y valorización de los ideales, la filosofía no deberá ser jamás una elegancia pasiva ulterior sino una fuerza creadora inicial, un fecundo drama del espíritu frente al porvenir. (1947: 83)

Gracias a lo dicho anteriormente es que dice, a modo de apotegma, que: "el comienzo de una cultura latino-americana tiene que estar en el esfuerzo de un pensamiento filosófico creador, por más que los acontecimientos de éste no alcancen a ser enteramente originales" (1947: 87).

Por lo tanto, las fuerzas que guardaría todo pensamiento creador tienen estrecho vínculo con grupos humanos que el autor identifica en el inicial camino de formación y desarrollo y no se podría articular el juicio de una carencia de reflexión filosófica. La negación de este pensamiento vendría a ser un contrasentido si se postula que la filosofía más vigorosa responde a

11 Para Arturo Andrés Roig, "un filosofar matutino o auroral, confiere al sujeto una participación creadora y transformadora, en cuanto que la filosofía no es ejercida como una función justificatoria de un pasado, sino de denuncia de un presente y de anuncio de un futuro, abiertos a la alteridad como factor de real presencia dentro del proceso histórico de las relaciones humanas" (1981: 15). 
los momentos de plena formación de las sociedades. Por eso es que Pérez Reinoso no toma en cuenta los juicios sobre la imposibilidad de una filosofía propiamente latinoamericana, independiente de que esta no tenga un cariz de completa originalidad. Que, por cierto, no tendría sentido alguno. Esta idea es reflejada de la siguiente manera en palabras del peruano:

\begin{abstract}
Nada más falso, si la más pura y más fuerte filosofía se encuentra en todo pórtico, que afirmar que en la América Latina no puede haber todavía pensamiento filosófico propio ni una producción filosófica expresiva de su espíritu, dando como razón que sus pueblos son demasiado jóvenes y no han alcanzado un completo desarrollo intelectual [...] Precisamente porque somos demasiado jóvenes (si todavía lo somos después de siglo y cuarto de repetirnos la misma frase, ya que carecemos de edad sociológica) debemos guardar y defender el caudal de fuerzas bárbaras que poseemos y edificar sobre ellas, sin el trámite y el barniz de revisadas culturas de ropero, nuestros sentimientos y concepciones de la vida. (1947: 87-88)
\end{abstract}

Como dijimos anteriormente, una historia dirigida debe dar cuenta de la autoconciencia que deben tener los intelectuales latinoamericanos de su realidad, de sus posibilidades y de las aptitudes y particularidades que cuenta su sociedad para construir un pensamiento que, en vez de repetir, asimilar y construir desde lo que se nos dice desde afuera, sea capaz de encauzar todo un mover que acaece por los bordes de las sociedades más europeizadas de nuestra América. Así, nuestro pensamiento cogería la fuerza necesaria para levantar continuidades históricas con coherencia, propias de todo pensamiento que reflexiona acorde a sus circunstancias:

La vida telúrica de las grandes masas latino-americanas, dirigida y sublimizada por el trabajo del espíritu, ciertamente irá más allá de la obra esporádica y débil del saltimbanqui intelectual que desprecia su medio por su ineptitud para comprenderlo y elevarlo. (1947: 91)

Ramiro Pérez Reinoso nos persuade a apuntar los esfuerzos a un pensamiento latinoamericano de raíz auténtica, es más, visualiza un camino posible levantando reflexiones que emanen de las realidades americanas más alejadas del mundo urbano, o bien que las reflexiones se realicen en espacios occidentalmente eruditos, pero en clave "bárbara". Para poder estipular aquello, 
nuestro autor establece una serie de procedimientos, parámetros y límites que ayudarían a la creación de pensamiento en franco "desprendimiento" del mundo occidental. Para él, esta es una de las más importantes tareas que debe realizar todo pensador que quiere comprometerse con el futuro latinoamericano:

\begin{abstract}
Tarea valiosísima es, en consecuencia, para los latino-americanos dar el golpe de timón y ponerse fuera del derrotero de la nave europea. Otros cielos, otras atmósferas, otras aguas, otras tripulaciones y otros puertos, sin que esto signifique la oposición y la lucha contra los buenos regazos maternales que incubaron nuestro espíritu y le dieron grandes y valiosos instintos. Sólo ambicionamos nuestra ciudadanía histórica y nuestra libertad y responsabilidad para ser los autores de nuestro propio destino, saborear el fruto perfumado de nuestra tierra y ver surgir nuestra estirpe demótica. (1947: 104)
\end{abstract}

La orientación hacia en pensamiento situado es el que nos dará la clave para poder leer América Latina desde ojos no occidentales, pues en la realidad misma estarían todos los insumos necesarios para levantar una filosofía que acuñe sus propias conceptualizaciones, sin tener para ello que pedir prestado para una astuta asimilación y así leer nuestra realidad:

Lo que puede decirse en un país latino-americano, por lo tanto, no puede decirse con finalidades iguales en la Europa occidental y central. En ésta la revolución de la filosofía tiene que producirse paralelamente a la revolución del espíritu social. El nuevo élan de la multitud influirá, sin duda, en el espíritu de los filósofos y a su vez la obra espiritual de los filósofos tendrá ecos profundos en la nueva alma de las naciones. (1947: 99)

Para leer nuestra realidad filosóficamente, Pérez Reinoso insiste con las ideas de «autonomía intelectual», pues imperativo es mirar nuestra relación con la vieja Europa como una fase superada: "Será el paso decisivo en nuestra deserción del compás europeo. Muy pronto debemos mirar a Europa sólo con la simpatía estética, con la emoción curiosa con que Europa miró a su Oriente materno en el siglo XIX" (1947: 116). Estas ideas son repetitivas en el filósofo peruano, pues para él es tarea urgente entender que Europa con su bagaje cultural nos ha embobado a tal punto que no somos capaces de pensar con nuestra cuenta, creyendo así que la cultura latinoamericana debe ser una suerte de sucursal pobre del cauce intelectual europeo: 
Lo censurable es creer que sólo Europa tiene la clave de los destinos del mundo y que hay que continuar bajo el fatalismo de creernos una provincia espiritual europea y de seguir la suerte del carro extranjero. Debemos reaccionar activamente contra esta confesión de inferioridad. (1947: 136)

En conclusión, el mensaje es claro, no es que se desdeñe el pensamiento europeo, en absoluto, pero, como diría Martí: la clave está en crear. Para buscar dicha creación de tintes propios, tal como ya lo hemos indicado, Pérez Reinoso encuentra la potencialidad en las zonas menos urbanizadas de nuestra América; teniendo a las urbes como focos de occidentalización intensa. Reinoso es consciente que cada país debe vivir su particular proceso de autonomía debido a la heterogeneidad estructural que posee el continente; además, hay países más idóneos para dar marcha a este nuevo inicio en el pensamiento latinoamericano.

Siguiendo la idea anterior, Pérez Reinoso divide la cultura latinoamericana en tres sectores que representarían, a grandes rasgos, tres realidades simultáneas. Primero tenemos la América central y las Antillas, luego los países que denomina bolivarianos y, finalmente, la región atlántica del sur más Chile. Las Antillas y la América central serían un espacio poco propicio para el desarrollo de un pensamiento original, pues este sector está "demasiado cerca del carro norteamericano y son partículas demasiado pequeñas para no sentir el vértigo de la civilización nórdica" (1947: 137). En cuanto a Uruguay, Argentina, el Brasil meridional y Chile central y sureño, ellos vendrían a ser "la Magna Grecia latino-americana. Ahí se ha insertado Europa con más hondura y la suerte de Europa pesará ahí por mucho tiempo sobre los espíritus" (1947: 137). Por último, tenemos los países "bolivarianos" que incluirán a todos los países que se encuentran en el "corazón" del continente americano: Bolivia, Ecuador, Perú, Venezuela entre otros. Se incluye a este grupo de países a México ya que para nuestro autor "a pesar de su posición geográfica - México-, se encuentra sociológicamente en el grupo de los países bolivarianos" (1947: 137). En referencia a este sector del continente señala: 
La América bolivariana, situada en el centro, ofrece, pues, — aún geográficamentelas condiciones más favorables para ser la representativa de un espíritu continental y la cuna de un movimiento consciente e inteligente hacia nuestra propia cultura. Las ventajas sociológicas del mundo bolivariano son mayores para un pensamiento propiamente latino-americano, pero sus condiciones espirituales de hoy no ofrecen la riqueza y entrenamiento necesarios para la cristalización de corrientes espirituales americanistas. Mas, esas condiciones espirituales pueden llenarse dentro de pocas generaciones. (1947: 137-138)

Siguiendo esta lógica, si bien habría una realidad en y desde la cual generar pensamiento filosófico, habría carencia de "madurez espiritual" para poder desarrollarlo. Aquí es donde entra en juego el rol del intelectual como pieza importante para el desarrollo espiritual del continente. Pues de ellos sería la responsabilidad de generar todo un proyecto cultural americano; proyecto ambicioso el de Pérez Reinoso, arielísitico quizá, pues los intelectuales pasarían a generar una suerte de "aristocratismo intelectual" que desarrollaría las bases del devenir histórico-cultural del continente, lo cual, por ejemplo, daría que:

Por primera vez en el mundo moderno una ética no será elaborada consuetudinariamente por la sociedad misma sino voluntariamente para ella. La dirección de la historia futura deberá hacerse a base de una ética de valores precisos. Esa ética latino-americana de dirigentes se extenderá al pueblo con valor religioso y exigirá la transformación de todo otro credo tradicional. (1947: 142)

Si bien valoramos las buenas intenciones del autor, estimamos difícil seguir un camino en donde la democracia, el disenso y las negociaciones con la diferencia, no se hagan parte de la construcción de futuro. Sin embargo, consideramos interesante los esfuerzos que Pérez Reinoso realiza en, por ejemplo, levantar actores y voces que podríamos considerar, desde cierta perspectiva, subalternas y desde ahí iniciar un proyecto emancipador. Más aun considerando que en ese tiempo, en la filosofía institucionalizada del país, las aproximaciones al planteamiento latinoamericano pocas veces integraban a los indígenas u otros «subalternos» en sus discursos. Es por eso que consideramos a Pérez Reinoso como una figura de quiebre en esta filosofía. Como hemos 
mencionado anteriormente, nuestro autor fue una figura relevante en el proceso de institucionalización de la filosofía en el país: fue secretario de difusión en la Sociedad Chilena de Filosofía, dictaba clases y conferencias en la Universidad de Chile, escribía en la revista Atenea, entre otras cosas. No obstante, sus planteamientos poco eco produjeron. Frente a una historización de la filosofía institucionalizada chilena, y siguiendo a Arturo Andrés Roig, podemos decir que Ramiro Pérez Reinoso vendría a ser el sujeto de una historia «episódica» que, por cierto, genera trizaduras en la idea de continuidad propia de toda historia «hegemónica» ${ }^{12}$ (Roig, 2008).

Considerar a los sujetos que habitan las zonas más alejadas de las urbes tan importantes como a los hombres y mujeres de intensa labor filosófica de tradición occidental, queviven las urbes latinoamericanas, no es cosa habitual en la tradición filosófica chilena de aquel entonces. Sin embargo, y es un punto interesante para trabajar, no es que estuviera del todo ausente, ya que Clarence Finlayson ${ }^{13}$ articula ciertas ideas que podrían conectar con el proyecto de nuestro autor. No obstante, no vemos a otro representante chileno que escriba en tono similar en la época.

\section{PALABRAS FINALES Y PREGUNTAS ABIERTAS}

Para finalizar y retomando lo dicho al comienzo, la inspiración e intención de rescatar el pensamiento de Pérez Reinoso no es solo por una orientación en el marco historiográfico propio de la historia de las ideas, sino también, porque las reflexiones analizadas en este artículo fueron elaboradas y publicadas en el contexto de institucionalización de la filosofía en Chile.

12 En relación a lo mencionado, y siguiendo a Antonio Gramsci, Arturo Andrés Roig nos dice que "frente a la contraposición entre la "historia oficial", periódica y por cierto continua y la "historia" de los sectores marginados respecto del poder que no gozan del ocio que necesitan los historiadores para su labor, historia que se reduce a momentos puntuales que quedan señalados como rupturas sin significado, momentos de "irracionalidad" que no encajan dentro de una "racionalidad" que podría justificarlos" (Roig, 2008: 138).

13 Ver "Expresión de la cultura americana" y "Consideraciones acerca de la cultura filosófica en la América latina", ambos trabajos en Finlayson, C. (1969). Antología. Santiago: Editorial Andrés Bello. 
Creemos que esto tiene suma importancia ya que, a diferencia de lo que se cree, en ese momento se está discutiendo sobre la filosofía y la cultura latinoamericana en dicho país, lo que refuerza la importancia de rescatar pensamientos que pueden ser catalogados como «episódicos», como señalaría Roig, dentro del «ecosistema intelectual» de Chile.

Por ejemplo, Enrique Molina ${ }^{14}$ se aproxima a la cultura latinoamericana, con ciertos rasgos característicos y con una visión particular. Así mismo, Jorge Millas en sus primeros textos "Carta a Ortega y Gasset" e "Idea de la Individualidad", ${ }^{15}$ sobre todo en la introducción y conclusión del último, tiene una preocupación esencial sobre el desarrollo de la filosofía en Chile, identificando a nuestro país con ciertas características como la sobriedad para diferenciarse de otros países más tropicales:

El caso de Chile es de una precocidad de este tipo. Entre los países de América, se distingue por ciertos rasgos propios de la plenitud histórica, que sólo a través de varios siglos pueden consolidarse. El fundamental de todos estos rasgos a que aludo, el que regimenta a los demás, imponiéndoles su sello, es, sin duda, el de la sobriedad espiritual. Chile es un pueblo sobrio. Esta sobriedad suya, como que está en contraste con otros caracteres pueriles de su imagen histórica, es una anticipación de la que ha de ser, sin duda, su personalidad definitiva en sus tiempos de sazón [...] Lo contrario de sobriedad es frenesí, o, como debería decirse en América, tropicalismo. En virtud de un sinnúmero de razones geográficas, históricas, raciales, culturales -que las hay en los órdenes más diversos-, los chilenos ponen en sus cosas siempre la fuerza adecuada para el efecto justo. (2009: 26-27)

En conclusión, lo que diferencia a Reinoso de ambas posturases la centralidad que le otorga a lo bárbaro por sobre lo civilizado. Más allá de un

14 Ahumada, A., \& Vrsalovic, S. (2015). Las reflexiones de Enrique Molina en el pensamiento latinoamericano del período "entreguerras": su visión del continente americano. Revista Solar, 11(2), 91-110.

15 Para revisar en mayor profundidad este período de Jorge Millas, consultar las tesis: Ahumada, A. (2017). Ideas de América Latina y de Chile en el período de "fundación" y "normalización" de la filosofia en Chile: el caso de Enrique Molina Garmendia. (Tesis de Magister en Estudios Latinoamericanos). Universidad de Chile, Santiago, Chile; Vrsalovic, S. (2019). Re-pensando la figura del filósofo latinoamericano desde Jorge Millas y Juan Rivano. (Tesis de Doctorado en Filosofía). Universidad de Chile, Santiago, Chile. 
indigenismo esencialista, ${ }^{16}$ Reinoso plantea una riqueza cultural en las personas cercanas a la naturaleza, oponiéndose a las personas de la urbe. Esta miradano permite catalogar a Pérez Reinoso como un pensador que aportó al desarrollo filosófico del país, pero sí como un filósofo que se animó a pensar desde un lugar que aún no era reconocido como un espacio propiamente filosófico.

\section{REFERENCIAS}

Ahumada, A. (2017). Ideas de América Latina y de Chile en el período de "fundación" y "normalización" de la filosofía en Chile: el caso de Enrique Molina Garmendia (Tesis de Magister). Universidad de Chile. Santiago.

---. (2018). "Normalización filosófica: entre filosofía y cultura". Revista de la Academia; vol. 25. Disponible en: https://bit.ly/2FjilqEAhumada, A., y Vrsalovic, S. (2012). "La reflexiones de Enrique Molina en el pensamiento latinoamericano del periodo de "entreguerras": su visión del continente americano". SOLAR. Revista de Filosofía Iberoamericana, 91-110.

Dussel, E. (1994). 1942 El encubrimiento del otro. Hacia el origen del "mito de la modernidad”. La Paz: Plural editores - UMSA.

Finlayson, C. (1969). Antología. Santiago: Editorial Andrés Bello

Kutipa, W. (2014). Literatura y política: El caso de Carlos A. González Marín y la generación tacneña de los años veinte.Tacna: Editorial KOREKHENKE.

Martí, J. (1986). "Nuestra América". En VV.AA., Ideas en Torno de Latinoamérica (122-129). México: UNAM-UDUAL.

Montiel, E. (2000) Humanismo Americano. Lima: FCE

Obando, O. (2015). "La filosofía de la filosofía en el Perú en el siglo XX” En Pensamiento e Ideas. $\mathrm{N}^{\circ} 7,11-20$.

16 Ver Montiel, E. (2000) Humanismo Americano. Lima: FCE. 
Pérez Reinoso, R. (1924) “Definición del arte último". En Novecientos, №3, Junio, 5-7.

---. (1924) "Futuración”. En Novecientos, N5, agosto, 28.

---. (1925) "La realidad cósmica de la libertad". En Mercurio Peruano, Año $5, \mathrm{~N}^{\circ} 85 / 86,285-290$

---. (1926) “Reseña a Mariano Ibérico Rodríguez.” En Libros y Revistas, N³, pp. 34-35.

---. (1927) “Los miserables de Dostoievski” En El Mercurio Peruano, Año 10, $\mathrm{N}^{\circ} 113-114,418-424$.

---. (1928) "Páginas acerca de Gonzáles Prada" En Orientación, № 9, s/p.

---. (1931). Ideas para una concepción histórica de la filosofia. Lima: Lux Editores.

---. (1932). "El problema de la autonomía espiritual de la América Latina". Atenea, 120-132.

---. (1947). Mensaje sobre el destino de la cultura en América Latina. Santiago: Editorial Pax-Chile.

Quijano, A. (2000). "Colonialidad del poder, eurocentrismo y América Latina". En E. Lander (coord.), La colonialidad del saber: eurocentrismo y ciencias sociales, 201-246. Buenos Aires: CLACSO.

Roig, A. A. (1981). Teoría y crítica del pensamiento latinoamericano. México: Fondo de Cultura Económica.

---. (2008). El pensamiento latinoamericano y su aventura. Buenos Aires: Ediciones El Andariego.

Salazar Bondy, A. (1967). La filosofía en Perú: panorama histórico. Lima: Editorial Universo.

Sobrevilla, D. (1996). La filosofía contemporánea en el Perú: estudios, reseña y notas sobre su desarrollo y situación actual. Lima: Carlos Matta.

Vidal, S. (1949). "La Sociedad Chilena de Filosofía". Revista de Filosofia, $\mathrm{N}^{\circ}$ 1, 93-102. 
Vrsalovic Muñoz S. (2018), "Entre dos interpretaciones de la normalización de la filosofía: ¿apertura o cierre de la filosofía latinoamericana?”. En VV.AA., Filosofía e Historia de la Ciencia en el Cono Sur, 451-464. Córdoba: AFHIC.

---. (2019). Re-pensando la figura del filósofo latinoamericano desde Jorge Millas y Juan Rivano (tesis doctoral). Universidad de Chile. Santiago. 